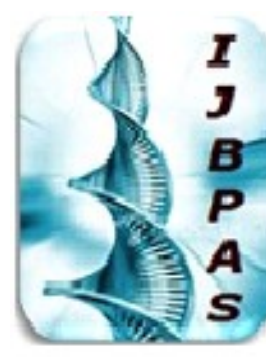

International Journal of Biology, Pharmaey and Allied Seiences (IJBPAS)

'A Bridge Betueen Caboratory and Q Q nador'

WwW.ibpas.com

SYNTHESIS, MOLECULAR DOCKING, AND BIOLOGICAL EVALUATION OF SOME NOVELINDOLE DERIVATIVES CONTAIN 1,3-THIAZOLE

\title{
SULTANA $\mathbf{S}^{1^{*}}$, PANDIAN $\mathbf{P}^{2}$ AND RAJKAMAL $\mathbf{B}^{3}$
}

1: Department of Pharmacy, K.V.K. College of Pharmacy, Hayathnagar-5015112 Hyderabad, Telangana, India

2: Department of Pharmacy, Annamalai University, Annamlainagar- 608002.

Chennai, Tamil Nadu, India

3: Department of Pharmacy, Malla Reddy Institute of Pharmaceutical Sciences. Hyderabad500100, Telangana, India

"Corresponding Authors: Shaheen Sulthana: Email: shaheen1314@gmail.com

Received $19^{\text {th }}$ May 2021; Revised $4^{\text {th }}$ June 2021; Accepted $9^{\text {th }}$ July 2021; Available online $25^{\text {th }}$ Sept. 2021 https://doi.org/10.31032/IJBPAS/2021/10.9.1019

\begin{abstract}
In present study, microwave assistedsynthesis of some novel Indole derivatives contain 1, 3thiazole was performed. All the synthesized products were purified through column chromatography and structures of these compounds were characterized by IR, ${ }^{1} \mathrm{H}$ NMR and mass spectral data. All the final derivatives were screened for their anticancer, antimicrobial activity and Molecular docking studies. All their efficacywas compared with standard drugs. The synthesized compound $\mathbf{4 b}, \mathbf{4 e}, \mathbf{4 i}$ and $\mathbf{4 j}$ are showed good Anticanceractivities whereas others exhibited significant activities. The compound $\mathbf{4 c}, \mathbf{4 d}, \mathbf{4 e}$ and $\mathbf{4 i}$ are has showed maximum antibacterial activity compare with streptomycin as a standard drug. The molecular docking studies of these novel derivatives of indole showed good agreement with the biological results when their binding pattern and affinity towards the active site of EGFR was investigate. Molecular docking study has been performed for all the synthesized compounds with MCFAcetylcholine-binding protein (AChBP) and results obtained are quite promising.
\end{abstract}

Keywords: Indole, 1,3-thiazole, Molecular docking, Anti-bacterial, Anticancer activity 


\section{INTRODUCTION}

The microwave assisted synthesis articulates an area of research, developing from scientific discoveries about pollution awareness and it exploits a set of principles that reduces or eliminates the generation of hazardous chemicals in all steps of synthetic process. Substituted 1,3-thiazole nucleus and Indole moiety has engaged a distinctive place in the field of medicinal chemistry due to widespread range of Pharmacological activities [1-3]. The Molecular design and the microwave synthesis of the bioactive nitrogen, sulphurcontaining heterocycles are at the top of the drug discovery and development. Substituted heterocyclic fragments constitute the core structure of a large number of pharmacologically interesting medicinal scaffolds present in numerous life-saving drugs [4-5]. Among these, substituted 1,3-thiazole nucleus and Indole skeletons are widely well used on designing several medicinal frameworks with a large number of Pharmacological belongings such as antimicrobial, anti-inflammatory, anticancer, antitubercular, and antiviral activities [6-9].

In conventional devices such as oil bath and heating mantles, are not only show for heating responses but also it produces a hot surface area on the reaction vessel. In microwave power alternatively is brought right into the chemical reactor from another location and travels through the walls of the response vessel, warming the reagents, catalysts, and solvents directly. Recently, a new technique such as microwave dielectric heating has actually concerned the leading edge of chemical investigation [10-12]. Microwaves assisted synthesis is a high-speed technic with has actually attracted a significant quantity of rate of interest in most recent years. The new scaffolds1,3-thiazole moiety contain novel Indole derivatives possessing latent functional substituents appear promising to fulfil the objectives of our Pharmacological activities and the desired chemical transformations.

\section{MATERIAL AND METHODS}

Melting points were measured by usingon open capillaries, using Toshniwal melting point apparatus, expressed in ${ }^{\circ} \mathrm{C}$ and are uncorrected. IR spectra of the compounds were recorded using on thermo Nicolet Nexus 670S series, FT-IR spectrometer using $\mathrm{KBr}$ disc. ${ }^{1} \mathrm{H}$ NMR was scanned on Avance-400 MHz instrument and chemical shifts are expressed in $\delta(\mathrm{ppm})$ relative to TMS as an internal standard using DMSO$d_{6}$ as solvent. The mass spectra were recorded on a LC-MSD-Trap-SL. The purity of the compounds was checked on silica gel-coated aluminium sheets (Merck, 1.005554, silica gel HF254-361, Type 60, $0.25 \mathrm{~mm}$ ) by analytical thin-layer 
chromatography (TLC). TLC was performed on silica gel G for TLC (Merck) and spots were visualized by iodine vapour or by irradiation with ultraviolet light (short w ave length, $254 \mathrm{~nm})$. Column chromatography was performed by using Qualigen's silica gel for column chromatography (60-120 mesh).

\subsection{General procedure [13-16]}

Step 1: Synthesis of 2-Amino 4-Aryl Thiazole (Cyclizisation reaction). A mixture of substituted acetophenone (0.1 $\mathrm{mol})$, thiourea $(0.2 \mathrm{~mol})$ and Iodine $(0.1$ mol) were placed in an open vessel containing a Teflon coated stir bar. The vessel was placed in the microwave cavity (CEM, Discover) and subjected to MW irradiation at $140^{\circ} \mathrm{C}$ for $10 \mathrm{~min}$. Then after the completion of the reaction crude mixture was cooled to $70^{\circ} \mathrm{C}$ and then it was triturated, filtered and washed with ethanol. The crude product was dissolved in hot water and the $\mathrm{pH}$ was adjusted to $11-12$ with $\mathrm{NH}_{4} \mathrm{OH}$. The precipitate was filtered and crystallized from mixture of ethanol: water (1:4) to get the product.

\section{Step: $\quad 2 . \quad 1-(1 H-i n d o l-3-y l)-N-(4-$ phenylthiazol-2-yl) methanimine (2a-} $2 g$ ).To a solution of equimolar quantity of compound (1a-1g) (0.01 mol) and Indole-3carboxaldehyde $(0.01 \mathrm{~mol})$ was added into absolute ethanol $(20 \mathrm{ml})$ and $10 \mathrm{ml}$ of glacial acetic acid, then MW irradiation 300Wfor 8-10 min. Then after the completion of the reaction mixture was cooled to room temperature and the solvent was then distilled off and the product obtained was recrystallized using ethanol. The progress of the reaction was monitored by TLC (nHexane: Ethyl acetate 7:3).

Step: 3: Preparation of $(\mathrm{N}-(1,3-$ benzothiazol-2-yl)-2-chloroacetamide)

$(3 a-3 b)$.To a solution of 2-amino benzothiazole $(0.016 \mathrm{~mol})$ in $(30 \mathrm{ml})$ glacial acetic acid, chloroacetyl chloride $(3.7 \mathrm{~g}$, $0.032 \mathrm{~mol}$ ) was added drop wise with constant stirring. The reaction mixture wasMW irradiation $150 \mathrm{~W}$ for 3-5 min, then it was powered in to crushed ice. The precipitated solid that obtained was filtered off, washed with cold water, dried and recrystallized from aqueous ethanol.

Step:4. N-(benzo[d]thiazol-2-yl)-2-(3-(((4phenylthiazol-2-yl) imino) methyl)-1Hindol-1-yl) acetamide (4a-4l). To a solution of Compound $(2 \mathrm{a}-2 \mathrm{~g})(0.016 \mathrm{~mol})$ in $(30 \mathrm{ml})$ glacial acetic acid, N-(benzo[d]thiazol-2yl)-2-chloroacetamide (3a-3b) (0.032mol) was added drop wise with constant stirring. The reaction mixture wasMW irradiation $150 \mathrm{~W}$ for $3-5 \mathrm{~min}$, then it was powered in to crushed ice. Then it was powered onto crushed ice. The precipitated solid that obtained was filtered off, washed with cold water, dried and recrystallized from aqueous ethanol. 
2.2.Physical and Spectral data of the compounds:

4a: $\quad N$-(benzo[d]thiazol-2-yl)-2-(3-(((4phenylthiazol-2-yl)imino)methyl)-1Hindol-1-yl) acetamide:M.P. 187$189^{\circ} \mathrm{C}$;Mol. formula: $\mathrm{C}_{27} \mathrm{H}_{19} \mathrm{~N}_{5} \mathrm{OS}_{2}$, yield 78\%, IR $(v \mathrm{~cm}-1): 3414(\mathrm{NH}$ Str, -CO-NH), 3062(C-H Str, Ar), 2992, 2842(C-H Str, Aliphatic), 2349 (C-S-C Str, Thazole), 1700(C=O Str, $-\mathrm{CO}-\mathrm{NH}-), 1636(\mathrm{C}=\mathrm{N}$ Str $)$, $1548(\mathrm{C}=\mathrm{CH} \quad$ Str $), \quad 1484(\mathrm{C}=\mathrm{C} \quad$ Str, Ar $)$, 892(C-N Str). ${ }^{1} \mathrm{H}-\mathrm{NMR}$ (DMSO) $\delta \delta$ ppm: 11.513(s, 1H, Acetamide), 8.997(s, Immine proton, $\mathrm{CH}=\mathrm{N}), 8.371(\mathrm{~s}, 1 \mathrm{H}, \mathrm{Ar}-\mathrm{H}), 7.897$ 7.846(d, 2H, Ar-H), 7.798-7.780(d, 2H, ArH), 7.695-7.685(d, 2H, Ar-H), 7.601(s, 1H, Ar-H), 7.581-7.548(t, 2H, Ar-H), 7.4987.480(t, 2H, Ar-H), 4.971-4.818(s, 2H, $\mathrm{CH}_{2}-\mathrm{CO}$ ), 2.284-2.169(s, 1H, -CH-). Mass (LC-MS): m/z 493(M), 494(M + 1, 100\%).

4b: $\quad N-($ benzo[d]thiazol-2-yl)-2-(3-(((4-(ptolyl)thiazol-2-yl)imino)methyl)-1H-indol1-yl) acetamide: M.P. $203-205^{\circ} \mathrm{C}$;Mol. formula: $\mathrm{C}_{28} \mathrm{H}_{21} \mathrm{~N}_{5} \mathrm{OS}_{2}$, yield $84 \%$, IR ( $v$ cm-1): 3423(NH Str, -CO-NH-), 3012(CH Str, Ar), 2910, 2891, 2732(C-H Str, Aliphatic), 2323(C-S-C Str, Thazole), 1712(C=O Str, $-\mathrm{CO}-\mathrm{NH}-), 1648(\mathrm{C}=\mathrm{N}$ Str $)$, $1548(\mathrm{C}=\mathrm{CH}$ Str $), \quad 1485(\mathrm{C}=\mathrm{C} \quad$ Str,$\quad \mathrm{Ar})$, 972(C-N Str). ${ }^{1} \mathrm{H}-\mathrm{NMR}$ (DMSO) $\delta \delta$ ppm: 10.691(s, 1H, Acetamide), 8.893(s, Immine proton, $\mathrm{CH}=\mathrm{N}), 7.979(\mathrm{~s}, 1 \mathrm{H}, \mathrm{Ar}-\mathrm{H}), 7.809$ 7.783(d, 2H, Ar-H), 7.658-7.631(d, 2H, Ar-
H), 7.520(s, 1H, Ar-H), 7.485-7.473(d, 2H, Ar-H), 7.443(t, 2H, Ar-H), 7.581-7.433(d, 2H, Ar-H), 7.392-7.388(t, 2H, Ar-H), 4.744-4.733(s, 2H, $\left.-\mathrm{CH}_{2}-\mathrm{CO}\right), 2.185(\mathrm{~s}, 1 \mathrm{H}$, Ar- $\mathrm{CH}_{3}$ ). Mass (LC-MS): m/z 507(M), $508(\mathrm{M}+1,100 \%)$.

4c: $\quad N-($ benzo[d]thiazol-2-yl)-2-(3-(((4-(4chlorophenyl)thiazol-2-yl)imino)methyl)-

1H-indol-1-yl) acetamide:M.P. 254$256^{\circ} \mathrm{C}$;Mol. formula: $\mathrm{C}_{27} \mathrm{H}_{18} \mathrm{~N}_{5} \mathrm{OClS}_{2}$, yield 76\%, IR $(v \mathrm{~cm}-1): 3403(\mathrm{NH}$ Str, $-\mathrm{CO}-\mathrm{NH}-$ ), 3082(C-H Str, Ar), 2952, 2810(C-H Str, Aliphatic), 2351 (C-S-C Str, Thazole), 1712(C=O Str, $-\mathrm{CO}-\mathrm{NH}-), 1653(\mathrm{C}=\mathrm{N}$ Str $)$, 1532(C $=\mathrm{CH} \quad$ Str $), \quad 1405(\mathrm{C}=\mathrm{C} \quad$ Str, Ar $)$, 1083(C-N Str), 794(Cl Str, Ar-Cl). ${ }^{1} \mathrm{H}-$ NMR (DMSO) $\delta \delta$ ppm: 11.498(s, 1H, Acetamide), 8.916(s, Immine proton, $\mathrm{CH}=\mathrm{N})$, 7.978(s, 1H, Ar-H), 7.902-7.876(d, 2H, Ar-H), 7.848-7.795(d, 2H, Ar-H), 7.785(s, 1H, Ar-H), 7.695-7.686(d, 2H, Ar$\mathrm{H}), \quad 7.606-7.596(\mathrm{t}, \quad 2 \mathrm{H}, \quad \mathrm{Ar}-\mathrm{H}), \quad 7.583-$ 7.518(d, 2H, Ar-H), 7.491-7.488(t, 2H, Ar$\mathrm{H}), 4.769-4.768\left(\mathrm{~s}, 2 \mathrm{H},-\mathrm{CH}_{2}-\mathrm{CO}\right)$. Mass (LC-MS): m/z 527(M), 528(M + 1, 100\%), $529(\mathrm{M}+2,30 \%)$.

4d: $\quad$ N-(benzo[d]thiazol-2-yl)-2-(3-(((4-(4fluorophenyl) thiazol-2-yl)imino)methyl)1H-indol-1-yl) acetamide: M.P. 213215 ${ }^{\circ} \mathrm{C}$;Mol. formula: $\mathrm{C}_{27} \mathrm{H}_{18} \mathrm{~N}_{5} \mathrm{O}_{2} \mathrm{FS}_{2}$, yield 77\%, IR $(v \mathrm{~cm}-1): 3422(\mathrm{NH}$ Str, -CO-NH), 3010(C-H Str, Ar), 2991, 2842(C-H Str, Aliphatic), 2323 (C-S-C Str, Thazole), 
1713(C=O Str, $-\mathrm{CO}-\mathrm{NH}-), 1636(\mathrm{C}=\mathrm{N}$ Str $)$, $1542(\mathrm{C}=\mathrm{CH}$ Str $), \quad 1383(\mathrm{C}=\mathrm{C} \quad$ Str,$\quad \mathrm{Ar})$, 930(C-N Str), 851(F Str, Ar-F). ${ }^{1} \mathrm{H}-\mathrm{NMR}$ (DMSO) $\delta \delta$ ppm: 10.958(s, $1 \mathrm{H}$, Acetamide), 8.671(s, Immine proton, $\mathrm{CH}=\mathrm{N}), 8.105(\mathrm{~s}, 1 \mathrm{H}, \mathrm{Ar}-\mathrm{H}), 7.886-7.878(\mathrm{~d}$, 2H, Ar-H), 7.843(d, 2H, Ar-H), 7.6857.658(d, 2H, Ar-H), 7.638-7.581(t, 2H, ArH), 7.574-7.556(d，2H, Ar-H), 7.5167.146(t, 2H, Ar-H), 7.16(s, 1H, Ar-H), 4.567-4.565(s, 2H, - $\left.\mathrm{CH}_{2}-\mathrm{CO}\right)$. Mass (LCMS): $\mathrm{m} / \mathrm{z}$ 511(M), 512(M + 1, 100\%), 513(M+2, 30\%).

4e: $\quad \mathrm{N}-($ benzo[d]thiazol-2-yl)-2-(3-(((4-(4methoxyphenyl) thiazol-2-

\section{yl)imino)methyl)-1H-indol-1-yl)}

acetamide: M.P. $187-189^{\circ} \mathrm{C}$;Mol. formula: $\mathrm{C}_{27} \mathrm{H}_{19} \mathrm{~N}_{2} \mathrm{OS}$, yield $78 \%$, IR ( $v$ cm-1): 3414(NH Str, -CO-NH-), 3062(C-H Str, Ar), 2992, 2842(C-H Str, Aliphatic), 2349 (C-S-C Str, Thazole), 1700(C=O Str, -CO$\mathrm{NH}-), \quad 1636(\mathrm{C}=\mathrm{N}$ Str $), \quad 1548(\mathrm{C}=\mathrm{CH}$ Str $)$, 1484(C=C Str, Ar), 972(C-N Str), 809(Cl Str, Ar-Cl). ${ }^{1} \mathrm{H}-\mathrm{NMR}$ (DMSO) $\delta \delta$ ppm: 12.304(s, 1H, Acetamide), 9.839(s, Immine proton, $\mathrm{CH}=\mathrm{N}), 8.456(\mathrm{~s}, 1 \mathrm{H}, \mathrm{Ar}-\mathrm{H}), 8.183$ 8.058(d, 2H, Ar-H), 7.969-7.694(d, 2H, ArH), 7.591-7.534(d, 2H, Ar-H), 7.5257.5059(t, 2H, Ar-H), 7.5057(s, 1H, Ar-H), 7.4904-7.4900(d, 2H, Ar-H), 7.474-7.470(t, $2 \mathrm{H}$, Ar-H), 4.7951-4.7950(s, 2H, - $\mathrm{CH}_{2}-$ $\mathrm{CO}), \quad 3.795-3.794\left(\mathrm{~s}, 1 \mathrm{H},-\mathrm{OCH}_{3}\right)$. Mass (LC-MS): m/z 493(M), 494(M + 1, 100\%). 4f: N-(benzo[d]thiazol-2-yl)-2-(3-(((4-(4nitrophenyl) thiazol-2-yl)imino)methyl)1H-indol-1-yl) acetamide.M.P. 211$213^{\circ} \mathrm{C}$; Mol. formula: $\mathrm{C}_{24} \mathrm{H}_{18} \mathrm{~N}_{6} \mathrm{O}_{3} \mathrm{~S}_{2}$, yield 74\%, IR ( $v$ cm-1): 3402(NH Str, -CO-NH), 3054(C-H Str, Ar), 2987, 2865(C-H Str, Aliphatic), 2340(C-S-C Str, Thazole), $1702(\mathrm{C}=\mathrm{O} \quad$ Str, $\quad-\mathrm{CO}-\mathrm{NH}-), \quad 1632\left(\mathrm{NO}_{2}\right)$, 1621(C=N Str $), 1535(\mathrm{C}=\mathrm{CH}$ Str $) .{ }^{1} \mathrm{H}-\mathrm{NMR}$ (DMSO) $\delta \delta \quad \mathrm{ppm}: \quad 11.763(\mathrm{~s}, \quad 1 \mathrm{H}$, Acetamide), 8.671(s, Immine proton, $\mathrm{CH}=\mathrm{N}), 8.281(\mathrm{~s}, 1 \mathrm{H}, \mathrm{Ar}-\mathrm{H}), 7.763-7.673(\mathrm{~d}$, 2H, Ar-H), 7.434-7.402(d, 2H, Ar-H), 7.320-7.243(d, 2H, Ar-H), 7.102(s, 1H, ArH), 7.043-7.002(t, 2H, Ar-H), 6.8736.801(t, 2H, Ar-H), 4.893-4.801(s, 2H, $\left.\mathrm{CH}_{2}-\mathrm{CO}\right), 2.453-2.324(\mathrm{~s}, 1 \mathrm{H},-\mathrm{CH}-)$. Mass (LC-MS): $\mathrm{m} / \mathrm{z}$ 538.09(M), 539(M + 1, $100 \%)$.

4g: N-(benzo[d]thiazol-2-yl)-2-(3-(((4-(4ethylphenyl)thiazol-2-yl)imino)methyl)-

1H-indol-1-yl) acetamide. M.P. 201203 ${ }^{\circ} \mathrm{C}$;Mol. formula: $\mathrm{C}_{29} \mathrm{H}_{23} \mathrm{~N}_{5} \mathrm{OS}_{2}$, yield 78\%, IR $(v \mathrm{~cm}-1): 3401(\mathrm{NH}$ Str, -CO-NH), 3072(C-H Str, Ar), 2932, 2856, 2798(CH Str, Aliphatic), 2343(C-S-C Str, Thazole), $\quad 1723(\mathrm{C}=\mathrm{O} \quad$ Str,$\quad-\mathrm{CO}-\mathrm{NH}-)$, 1632(C=N Str $), 1532(\mathrm{C}=\mathrm{CH}$ Str $), 973(\mathrm{C}-\mathrm{N}$ Str). ${ }^{1} \mathrm{H}-\mathrm{NMR}$ (DMSO) $\delta \delta$ ppm: 11.532(s, 1H, Acetamide), 8.893(s, Immine proton, $\mathrm{CH}=\mathrm{N})$, 7.893(s, 1H, Ar-H), 7.789-7.698(d, 2H, Ar-H), 7.345-7.231(d, 2H, Ar-H), 7.291(s, 1H, Ar-H), 7.172-7.132(d, 2H, Ar- 
H), 7.101(t, 2H, Ar-H), 6.892-6.802(d, 2H, Ar-H), 6.543-6.211(t, 2H，Ar-H), 4.6734.592(s, 2H, $\left.-\mathrm{CH}_{2}-\mathrm{CO}\right), 2.234-2.201(\mathrm{~s}, 2 \mathrm{H}$, $\left.\mathrm{Ar}-\mathrm{CH}_{2-}\right) . \quad 2.012-2.002\left(\mathrm{~s}, \quad 1 \mathrm{H}, \mathrm{Ar}-\mathrm{CH}_{3}\right)$. Mass (LC-MS): m/z 521.13(M), 522(M + $1,100 \%)$.

4h: N-(6-methylbenzo[d]thiazol-2-yl)-2-(3(((4-phenylthiazol-2-yl)imino)methyl)-1Hindol-1-yl) acetamide. M.P. 183$185^{\circ} \mathrm{C}$;Mol. formula: $\mathrm{C}_{28} \mathrm{H}_{21} \mathrm{~N}_{5} \mathrm{OS}_{2}$, yield 81\%, IR $(v \mathrm{~cm}-1): 3412(\mathrm{NH}$ Str, -CO-NH), 3027(C-H Str, Ar), 2987, 2856(C-H Str, Aliphatic), 2345(C-S-C Str, Thazole), 1712(C=O Str, $-\mathrm{CO}-\mathrm{NH}-), 1626(\mathrm{C}=\mathrm{N}$ Str $)$, $1546(\mathrm{C}=\mathrm{CH} \quad$ Str $), \quad 1410(\mathrm{C}=\mathrm{C} \quad$ Str,$\quad \mathrm{Ar})$, 1021(C-N Str). ${ }^{1} \mathrm{H}-\mathrm{NMR}$ (DMSO) $\delta \delta$ ppm: 12.092(s, 1H, Acetamide), 8.938(s, Immine proton, $\mathrm{CH}=\mathrm{N}), 7.998(\mathrm{~s}, 1 \mathrm{H}, \mathrm{Ar}-\mathrm{H}), 7.890$ 7.783(d, 2H, Ar-H), 7.673-7.663(d, 2H, ArH), 7.543(s, 1H, Ar-H), 7.495-7.439(d, 2H, Ar-H), 7.236-7.201(t, 2H, Ar-H), 7.1037.012(d, 2H, Ar-H), 6.980(t, 2H, Ar-H), 4.854-4.802(s, 2H, - $\left.\mathrm{CH}_{2}-\mathrm{CO}\right)$. Mass (LCMS): m/z 507.12(M), 508(M+1, 100\%).

4i: $\quad$ 2-(3-(((4-(4-ethylphenyl)thiazol-2yl)imino)methyl)-1H-indol-1-yl)- $\mathrm{N}-(6-$ methyl benzo [d]thiazol-2-yl) acetamide. M.P. $\quad 173-175^{\circ} \mathrm{C}$;Mol. formula: $\mathrm{C}_{30} \mathrm{H}_{25} \mathrm{~N}_{5} \mathrm{OS}_{2}$, yield $76 \%$, IR ( $v$ cm-1): 3442(NH Str, -CO-NH-), 3072(C-H Str, Ar), 2967, 2867(C-H Str, Aliphatic), 2365(C-S-C Str, Thazole), 1722(C=O Str, CO-NH- $), \quad 1627(\mathrm{C}=\mathrm{N} \quad$ Str $), \quad 1532(\mathrm{C}=\mathrm{CH}$ Str $), 1398\left(\mathrm{C}=\mathrm{C}\right.$ Str, Ar). ${ }^{1} \mathrm{H}-\mathrm{NMR}$ (DMSO) $\delta \delta$ ppm: 12.053(s, 1H, Acetamide), 8.892(s, Immine proton, $\mathrm{CH}=\mathrm{N}), 8.321(\mathrm{~s}$, 1H, Ar-H), 8.092-8.002(d, 2H, Ar-H), 7.982(d, 2H, Ar-H), 7.873-7.783(d, 2H, Ar$\mathrm{H}), \quad 7.574-7.473(\mathrm{t}, \quad 2 \mathrm{H}, \quad$ Ar-H), 7.3427.301(d, 2H, Ar-H), 7.320-7.302(t, 2H, ArH), 7.201(s, 1H, Ar-H), 4.672-4.601(s, 2H, $\left.-\mathrm{CH}_{2}-\mathrm{CO}\right), 2.320\left(\mathrm{q}, 2 \mathrm{H},-\mathrm{CH}_{2}-\right), 2.021-$ 2.001(s, 2H, $\left.-\mathrm{CH}_{3}\right)$. Mass (LC-MS): $\mathrm{m} / \mathrm{z}$ 535(M), 536(M +1, 100\%).

\begin{tabular}{|c|c|c|c|c|c|c|}
\hline Code & $\mathbf{R}$ & $R_{1}$ & Mol. Formula & Mol. wt (g.mol ${ }^{-1}$ ) & M.P ( C) & $\mathrm{R}_{\mathrm{f}}$ Value \\
\hline $4 a$ & $-\mathrm{H}$ & $-\mathbf{H}$ & $\mathrm{C}_{27} \mathrm{H}_{19}^{\mathrm{N}} \mathrm{N}_{5} \mathrm{OS}_{2}$ & 493.10 & $187-189$ & 0.65 \\
\hline $4 b$ & $\mathrm{CH}_{3}$ & $-\mathrm{H}$ & $\mathrm{C}_{28} \mathrm{H}_{21} \mathrm{~N}_{5} \mathrm{OS}_{2}$ & 507.12 & $203-205$ & 0.77 \\
\hline $4 c$ & $-\mathrm{Cl}$ & $-\mathbf{H}$ & $\mathrm{C}_{27} \mathrm{H} \underset{18}{\mathrm{~N}} \mathrm{OClS}_{2}$ & 527.06 & $254-256$ & 0.63 \\
\hline 4d & $-F$ & $-\mathrm{H}$ & $\mathrm{C}_{27} \mathrm{H}_{18} \mathrm{~N}_{5} \mathrm{O}_{2} \mathrm{FS}_{2}$ & 511.09 & 213-215 & 0.71 \\
\hline $4 e$ & $-\mathrm{OCH}_{3}$ & $-\mathrm{H}$ & $\mathrm{C}_{28} \mathrm{H}_{21} \mathrm{~N}_{5} \mathrm{O}_{2} \mathrm{~S}_{2}$ & 523.11 & $167-169$ & 0.58 \\
\hline $4 f$ & $-\mathrm{NO}_{2}$ & $-\mathbf{H}$ & $\mathrm{C}_{24} \mathrm{H}_{18} \mathrm{~N}_{6} \mathrm{O}_{3} \mathrm{~S}_{2}$ & 538.09 & 211-213 & 0.63 \\
\hline $4 \mathrm{~g}$ & $-\mathrm{C}_{2} \mathrm{H}_{5}$ & $-\mathrm{H}$ & $\mathrm{C}_{29} \mathrm{H}_{23} \mathrm{~N}_{6} \mathrm{OS}_{2}$ & 521.13 & $201-203$ & 0.59 \\
\hline $4 h$ & $-\mathbf{H}$ & $-\mathrm{CH}_{3}$ & $\mathrm{C}_{28} \mathrm{H}_{21} \mathrm{~N}_{21} \mathrm{OS}_{2}$ & 507.12 & $183-185$ & 0.61 \\
\hline $4 i$ & $-\mathrm{C}_{2} \mathrm{H}_{5}$ & $-\mathrm{CH}_{3}$ & $\mathrm{C}_{30} \mathrm{H}_{25} \mathrm{~N}_{5} \mathrm{OS}_{2}$ & 535.15 & 173-175 & 0.79 \\
\hline $4 j$ & $-\mathrm{CH}_{3}$ & $-\mathrm{CH}_{3}$ & $\mathrm{C}_{29} \mathrm{H}_{23} \mathrm{~N}_{5} \mathrm{OS}_{2}$ & 521.13 & $223-225$ & 0.65 \\
\hline $4 k$ & $-\mathbf{F}$ & $-\mathrm{CH}_{3}$ & $\mathrm{C}_{28} \mathrm{H}_{20} \mathrm{~N}_{5} \mathrm{OFS}_{2}$ & 525.11 & $251-253$ & 0.70 \\
\hline 41 & $-\mathrm{OCH}_{3}$ & $-\mathrm{CH}_{3}$ & $\mathrm{C}_{29} \mathrm{H}_{23} \mathrm{~N}_{5} \mathrm{O}_{2} \mathrm{~S}_{2}$ & 537.13 & $177-179$ & 0.82 \\
\hline
\end{tabular}


4j: N-(6-methylbenzo[d]thiazol-2-yl)-2-(3(((4-(p-tolyl)thiazol-2-yl)imino)methyl)-

1H-indol -1-yl) acetamide.M.P. 223$225^{\circ} \mathrm{C}$; Mol. formula: $\mathrm{C}_{29} \mathrm{H}_{23} \mathrm{~N}_{2} \mathrm{OS}$, yield 75\%, IR $(v \mathrm{~cm}-1): 3421(\mathrm{NH} \mathrm{Str},-\mathrm{CO}-\mathrm{NH}-$ ), 3082(C-H Str, Ar), 2989, 2874(C-H Str, Aliphatic), 2332 (C-S-C Str, Thazole), 1723(C $=$ O Str, $-\mathrm{CO}-\mathrm{NH}-), 1628(\mathrm{C}=\mathrm{N}$ Str $)$, $1525(\mathrm{C}=\mathrm{CH} \quad$ Str $) .{ }^{1} \mathrm{H}-\mathrm{NMR} \quad(\mathrm{DMSO}) \quad \delta \delta$ ppm: 12.021(s, 1H, Acetamide), 8.909(s,
Immine proton, $\mathrm{CH}=\mathrm{N}), 8.372(\mathrm{~s}, 1 \mathrm{H}, \mathrm{Ar}-$ $\mathrm{H}), \quad 8.327-8.281(\mathrm{~d}, \quad 2 \mathrm{H}, \quad \mathrm{Ar}-\mathrm{H}), \quad 7.803-$ 7.801(d, 2H, Ar-H), 7.453-7.401(d, 2H, ArH), 7.320-7.301(t, 2H, Ar-H), 7.290(s, 1H, Ar-H), 7.203-7.201(d, 2H, Ar-H), 6.8736.820(t, 2H, Ar-H), 4.543-4.483(s, 2H, $\left.\mathrm{CH}_{2}-\mathrm{CO}\right), 2.063-2.003\left(\mathrm{~s}, 6 \mathrm{H},-\mathrm{CH}_{3}\right)$. Mass (LC-MS): $\mathrm{m} / \mathrm{z}$ 521.13(M), 522(M + 1, $100 \%)$.<smiles>[R]c1ccc(C(C)=O)cc1</smiles>

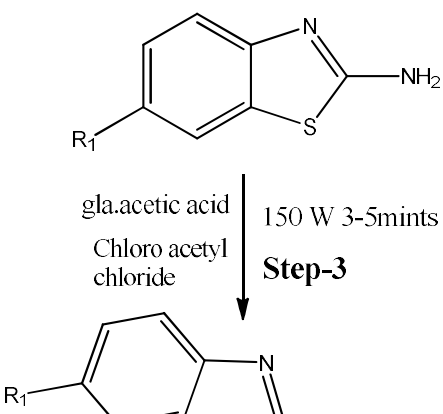

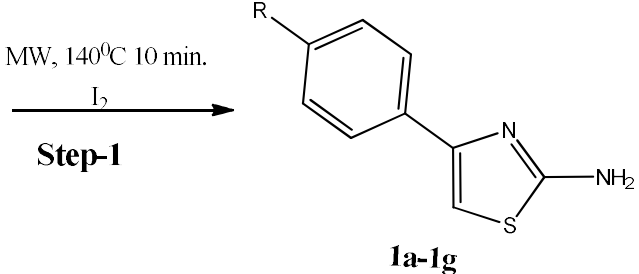
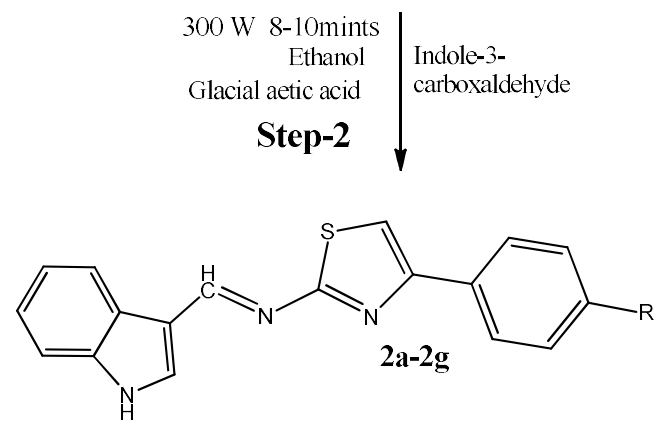

3a-3b<smiles>C/C=C\CC(C)C(C)C(C)C(=O)CCl</smiles>

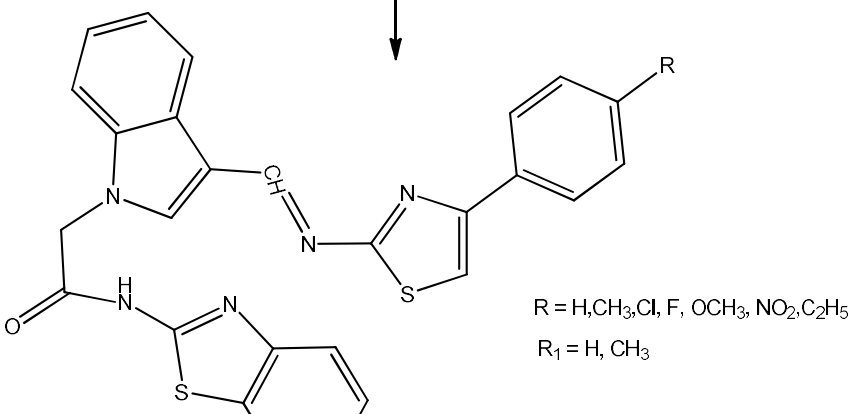

4a-4I

Figure 1: Scheme of synthesis for some novel Indole derivatives 
4k: 2-(3-(((4-(4-fluorophenyl) thiazol-2-yl) imino) methyl)-1H-indol-1-yl)-N-(6-methyl benzo[d]thiazol-2-yl) acetamide.M.P. 251$253^{\circ} \mathrm{C}$;Mol. formula: $\mathrm{C}_{28} \mathrm{H}_{20} \mathrm{~N}_{5} \mathrm{OFS}_{2}$, yield 72\%, IR $(v \mathrm{~cm}-1): \quad 3403(\mathrm{NH}$ Str, $-\mathrm{CO}-\mathrm{NH}-)$, 3035(C-H Str, Ar), 2969, 2833(C-H Str, Aliphatic), 2387(C-S-C Str, Thazole), $1705(\mathrm{C}=\mathrm{O} \quad$ Str,$\quad-\mathrm{CO}-\mathrm{NH}-), \quad 1628(\mathrm{C}=\mathrm{N} \quad$ Str $)$, 1532(C $=\mathrm{CH}$ Str $), 1354(\mathrm{C}=\mathrm{C}$ Str, Ar $), 867(\mathrm{~F}$ Str, Ar-F). $\quad{ }^{1} \mathrm{H}-\mathrm{NMR} \quad(\mathrm{DMSO}) \quad \delta \delta$ ppm: 11.932(s, 1H, Acetamide), 8.782(s, Immine proton, $\mathrm{CH}=\mathrm{N}), 8.231(\mathrm{~s}, 1 \mathrm{H}, \mathrm{Ar}-\mathrm{H}), 7.902-$ 7.900(d, 2H, Ar-H), 7.872(d, 2H, Ar-H), 7.5477.582(d, 2H, Ar-H), 7.434-7.403(t, 2H, Ar-H), 7.321-7.300(d, 2H, Ar-H), 7.219-7.201(t, 2H, Ar-H), 7.031(s, 1H, Ar-H), 4.347-4.286(s, 2H, $\mathrm{CH}_{2}-\mathrm{CO}$ ), 2.043-2.001(s, 3H, - $\mathrm{CH}_{3}$ ). Mass (LCMS): $\mathrm{m} / \mathrm{z}$ 525.11(M), 526(M + 1, 100\%), $527(\mathrm{M}+2,30 \%)$.

4l: 2-(3-(((4-(4-methoxyphenyl) thiazol-2yl)imino)methyl)-1H-indol-1-yl)-N-(6-methyl benzo [d]thiazol-2-yl)acetamide.M.P. 177$179^{\circ} \mathrm{C}$;Mol. formula: $\mathrm{C}_{29} \mathrm{H}_{23} \mathrm{~N}_{5} \mathrm{O}_{2} \mathrm{~S}_{2}$, yield $80 \%$, IR $(v \quad \mathrm{~cm}-1): \quad 3428(\mathrm{NH} \quad$ Str, $-\mathrm{CO}-\mathrm{NH}-)$, 3087(C-H Str, Ar), 2984, 2873(C-H Str, Aliphatic), 2332 (C-S-C Str, Thazole), $1716(\mathrm{C}=\mathrm{O} \quad$ Str, $\quad-\mathrm{CO}-\mathrm{NH}-), \quad 1621(\mathrm{C}=\mathrm{N} \quad$ Str $)$, 1543(C=CH Str), 1482(C=C Str, Ar), 998(C-N Str). ${ }^{1} \mathrm{H}-\mathrm{NMR}$ (DMSO) $\delta \delta$ ppm: 12.432(s, $1 \mathrm{H}$, Acetamide), 9.865(s, Immine proton, $\mathrm{CH}=\mathrm{N})$, 8.542(s, 1H, Ar-H), 8.281-8.201(d, 2H, Ar-H), 7.832-7.802(d, 2H, Ar-H), 7.656-7.632(d, 2H, Ar-H), 7.473-7.422(t, 2H, Ar-H), 7.320(s, 1H, Ar-H), 7.281-7.201(d, 2H, Ar-H), 7.1097.001(t, 2H, Ar-H), 4.832-4.802(s, 2H, $-\mathrm{CH}_{2}-$ $\mathrm{CO}), \quad 3.810-3.802\left(\mathrm{~s}, \quad 1 \mathrm{H}, \quad-\mathrm{OCH}_{3}\right), \quad 1.899-$ 1.802(s, 3H, $\left.-\mathrm{CH}_{3}\right)$. Mass (LC-MS): $\mathrm{m} / \mathrm{z}$ 537.13(M), 538(M+1,100\%).

\subsection{Pharmacological Evaluation:}

2.3.1. Anti-bacterial-activity [18]: All the novel synthesized compounds $(4 a-41)$ were evolved for their antibacterial activity against gram-positive strains such as Bacillus substillus, and Staphylococcus aureus, were as gram-negative strains such as Escherichia coli and Salmonella paratyphi species (Table 2) by using the agar disc cup plate diffusion method.All the synthesized compounds were dissolved in Ethanol and methanol (2:8) to get asolution of $1 \mathrm{mg} \mathrm{mL}-1$. Streptomycin used as a standard drug.After incubation period the zone of inhibition was measured in millimetresfor sample and standard drugs.Controlled solution alone showed no inhibition zone.

2.3.2. Anticancer activity [19]: The anticancer activity of synthesized compounds was determining in vitro by MTT Assay, andCell viability was once evaluated with three experiments with six concentrationsin triplicates. All test compounds were weighed separately and dissolved in DMSO and with media make up the final concentration to $1 \mathrm{mg} / \mathrm{ml}$ and the cells were treated with series of concentrations from $05,10,15,25,50,75$ and $100 \mu \mathrm{g} /$ ml.Cells were counted by haemocytometer and seeded at density of $5.0 \times 10^{3}$ cells / well in $100 \mu$ media in 96 well plate 
culture medium and incubated overnight at $37^{0}$ C.At the end of incubation time, precipitates are formed as a result of the reduction of the MTT salt to chromophore formazan crystals by the cells with metabolically active mitochondria. The optical density of solubilized crystals in DMSO was measured at $570 \mathrm{~nm}$ on a microplate reader. The percentage growth inhibition was calculated using the following formula (Table 2).

$$
\% \text { Inhibition }=\frac{100(\text { Control }- \text { Treatment })}{\text { Control }}
$$

The Ic50 value was determined by using linear regression equation i.e. $y=m x+c$. Here, $y=50, m$ and $c$ values were derived from the viability graph.

2.3.3. Molecular docking [20]: The binding of novel synthesized indole derivatives to aromatase was investigated in a docking procedure by applying a combined quantum mechanical/ molecular mechanical method. 2D structures of the compounds were converted to $3 \mathrm{D}$ using potential algorithms and application of high efficient force fields. Initial geometrical optimization and energy minimization of molecules were performed by using the Ligprep tool of Schrodinger suite. The digital structure of the Acetylcholinebinding protein $(\mathrm{AChBP})$ was retrieved from the Protein databank website with PDB Id: 2ZJU and the structure was optimizedby deleting unbound water molecules which are over $1 \AA$, adding hydrogen atoms to satisfy the valences, adding missing amino acids to stabilize side chains and energy of the whole structure was minimized using OPLS-2005 force field using Protein Preparation Wizard tool of Schrodinger Suite. Thus structurally optimized protein structure was used to examine protein-ligand interactions of the dataset ligands using Glide Xp docking protocol. Initially, a 3D grid was established to the binding pocket (active site) of the protein, into which all the dataset ligands were docked into. Binding interactions and efficiency of the binding were calculated in terms of Glide Score, which is a combination of hydrophilic, hydrophobic, metal binding groups, Van der Waals energy, freezing rotatable bonds and polar interactions with receptor.

\section{RESULTS AND DISCUSSION}

3.1. Chemistry:The protocol for all the synthesized novel indole derivatives is depicted in Scheme.In first step, a mixture of substituted acetophenone reacts with thiourea in the presence of Iodine to give 2amino 4-aryl thiazole, then it can be undergoing Schiff's base reaction with Indole-3-carboxaldehydeto produce $1-(1 \mathrm{H}-$ indol-3-yl)-N-(4-phenylthiazol-2-yl) methanimine (2a-2g). Were as in step-3, 2amino benzothiazole reacts with chloroacetyl chloride to give $(\mathrm{N}-(1,3-$ benzothiazol-2-yl)-2-chloroacetamide) (3a3b). Finally,3a-3g compounds reacts with $2 \mathrm{a}-2 \mathrm{~g}$ to give the title of the 
compounds.The chemical structures of all the synthesized compounds (4a-4l) were established by IR, NMR and Mass spectroscopic techniques and the yield of the compound ranges from $64 \%-83 \%$. The mass spectra of all the synthesized compounds (4a-4l) are in accordance with their molecular weights. Detailed spectral data is given in experimental protocols.

\subsection{Pharmacological Evaluation}

3.2.1. Antibacterial activity: Antibacterial activity of the synthesized compounds (4a4l) was compared with standard "Streptomycin" and the results are presented in Table 2. The most of the synthesized compounds like $\mathbf{4 c}, \mathbf{4 d}, \mathbf{4 f}$ and 4k are showed potent antibacterial activity against all the tested bacterial strains and $\mathbf{4 b}, \mathbf{4 e}, \mathbf{4 g}, \mathbf{4 i}$ and $4 \mathbf{l}$ are showed moderate antibacterial activity.

3.2.2. Anticancer activity: Novel Indole derivatives contain 1,3-thiazole were evaluatedfor cytotoxicity by MTT assay against human breast cancer cells (MCF7), and comparison with doxorubicin as standard. The results (Table 3, Figure 3) proposed that both cell lines were susceptible to the evaluated novel compounds. Compounds $4 \mathrm{a}-41$ showed IC50values in the range of $43.83 \mu \mathrm{M}$ to $84.44 \mu \mathrm{M}$ against MCF7 cell line. From the results, compounds $4 \mathbf{b}, 4 \mathbf{e}$ and $4 \mathbf{l}$ are the most active analogs in this study and showing broad spectrum activity toward the tested cell lines,whereas, remaining all other compounds showed moderate activity. All the test compounds results were shown in the table and expressed as a mean \pm SEM of five concentrations.

\subsubsection{Molecular docking studies:} Molecular docking studies were performed in order to find the possible protein ligand interactions of the dataset ligands. Additionally, these also assisted in identifying the conformational changes of the ligand in the protein environment. About generates 100 different protein ligand complex conformations for each docked complex were generated through Glide XP module. Based on the EModel energy, only one was displayed in the result. Glide dock sores of the dataset ligands were shown in Table 4, Figure 5, along with the interaction amino acids and number of amino acids.

Among the docked ligands, compound $4 \mathrm{c}$ reported highest dock score of -5.86 with Glide binding energy of $-38.599 \mathrm{Kcal} / \mathrm{mol}$. Compounds $4 \mathrm{c}, 4 \mathrm{l}$ and $4 \mathrm{k}$ possessed 3 hydrogen bonds, with Alanine 881 amino acid (2 bonds) and Glutamine 119 (1 bond. Dock scores of all the compounds ranged from -5.861 (compound 4c) to -2.261 (compound 4i). Nitrogen in the benzothiazole moiety and the amine group attached to the benzothiazole moiety are responsible for the $\mathrm{H}$-bond interaction. Hydrophobic interactions were identified 
between Tryptophan 143 amino acid and indole moiety of compounds $4 \mathrm{c}$ and $4 \mathrm{f}$. Indole moiety of compound $4 \mathrm{i}$ had hydrophobic interaction with Histidine 145.
Benzothiazole moiety of compounds $4 \mathrm{f}$ and $4 \mathrm{k}$ showed hydrophobic interactions with Tryptophan 143 and Tyrosine 185, respectively.

Table 2: Antibacterial activity of novel indole derivatives by zone of inhibition (in $\mathbf{~ m m}$ )

\begin{tabular}{|c|c|c|c|c|}
\hline \multirow[t]{2}{*}{ Compound } & \multicolumn{4}{|c|}{ Zone of Inhibition (in mm) } \\
\hline & B. substillus & St. aureus & E. coli & Salmonella paratyphi \\
\hline Streptomycin & 33 & 31 & 33 & 35 \\
\hline $4 \mathbf{a}$ & 18 & 22 & 09 & 18 \\
\hline $4 b$ & 25 & 23 & 21 & 09 \\
\hline $4 \mathrm{c}$ & 30 & 25 & 19 & 29 \\
\hline 4d & 29 & 28 & 28 & 26 \\
\hline $4 e$ & 28 & 26 & 22 & 23 \\
\hline 4f & 30 & 29 & 16 & 29 \\
\hline $4 \mathrm{~g}$ & 23 & 22 & 17 & 17 \\
\hline $4 h$ & 20 & 23 & 09 & 21 \\
\hline $4 i$ & 21 & 22 & 20 & 20 \\
\hline $4 j$ & 23 & 25 & 18 & 14 \\
\hline $4 k$ & 30 & 29 & 18 & 29 \\
\hline 41 & 25 & 22 & 28 & 19 \\
\hline
\end{tabular}

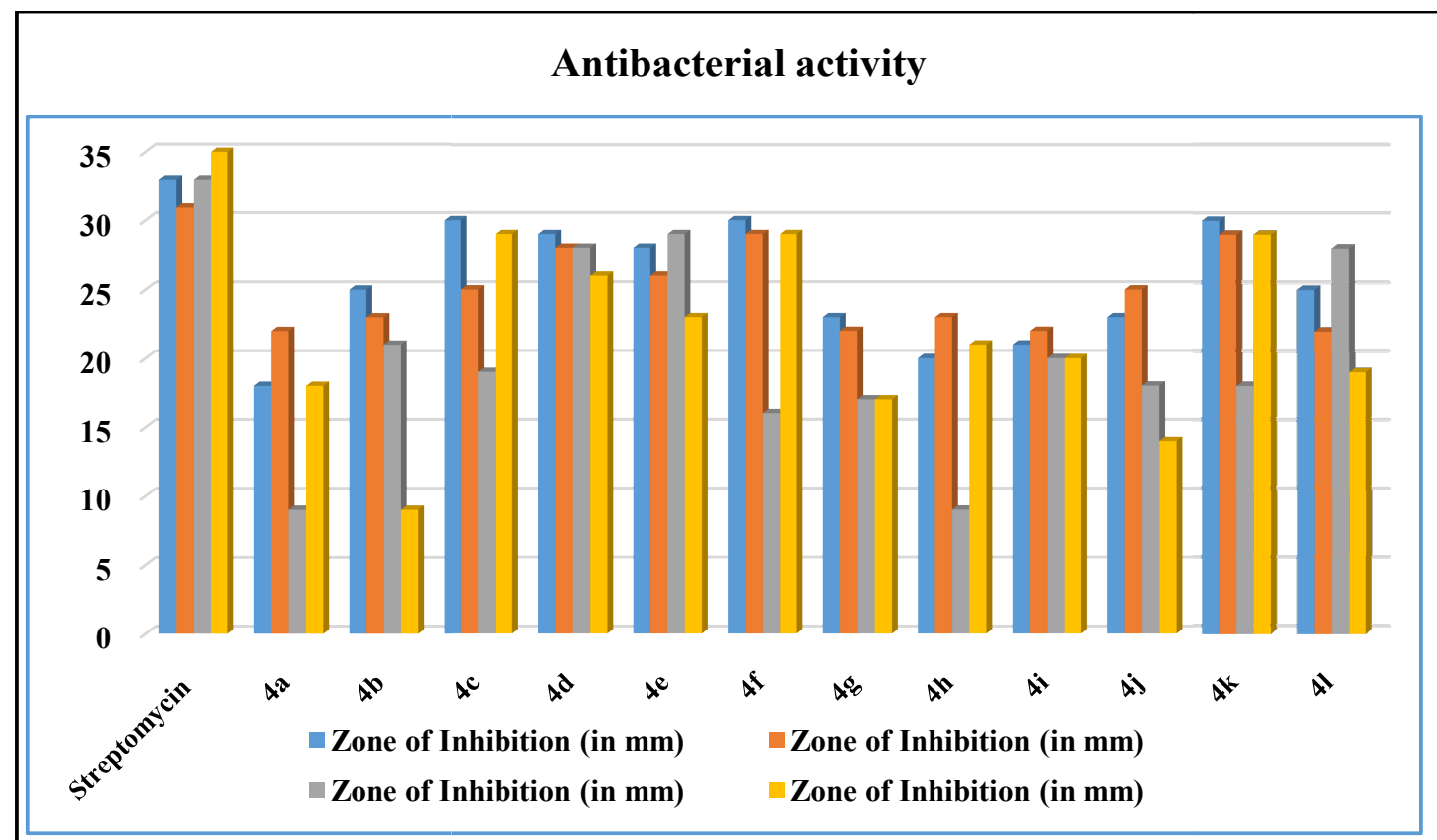

Figure 2: Graphical representation of antibacterial activity of novel indole compounds (4a-4l) - Zone of Inhibition (mm) 
Table 3: Cytotoxic Activity of novel indole derivatives on MCF-7 Cells

\begin{tabular}{|c|c|c|}
\hline S. No & Sample Name & IC $_{50}(\mu \mathrm{g})$ \\
\hline 1 & $4 \mathrm{a}$ & $\mathbf{8 3 . 2 1} \pm 0.321$ \\
\hline 2 & $4 \mathrm{~b}$ & $\mathbf{5 3 . 0 1} \pm 0.842$ \\
\hline 3 & $4 \mathrm{c}$ & $\mathbf{8 4 . 4 4} \pm 0.032$ \\
\hline 4 & $4 \mathrm{~d}$ & $\mathbf{8 8 . 2 1} \pm 0.002$ \\
\hline 5 & $4 \mathrm{e}$ & $\mathbf{5 1 . 0 4} \pm 0.631$ \\
\hline 6 & $4 \mathrm{f}$ & $\mathbf{4 9 . 0 3} \pm 0.031$ \\
\hline 7 & $4 \mathrm{~g}$ & $\mathbf{7 0 . 4 6} \pm 0.183$ \\
\hline 8 & $4 \mathrm{~h}$ & $\mathbf{5 8 . 3 2} \pm 0.031$ \\
\hline 9 & $4 \mathrm{i}$ & $\mathbf{9 0 . 2 3} \pm 0.005$ \\
\hline 10 & $4 \mathrm{j}$ & $\mathbf{7 0 . 3 2} \pm 0.103$ \\
\hline 11 & $4 \mathrm{k}$ & $\mathbf{6 9 . 9 2} \pm 0.204$ \\
\hline 12 & $4 \mathrm{l}$ & $\mathbf{4 3 . 8 3} \pm 0.702$ \\
\hline 13 & Doxorubicin & $\mathbf{1 5 . 4 3} \pm 0.142$ \\
\hline
\end{tabular}

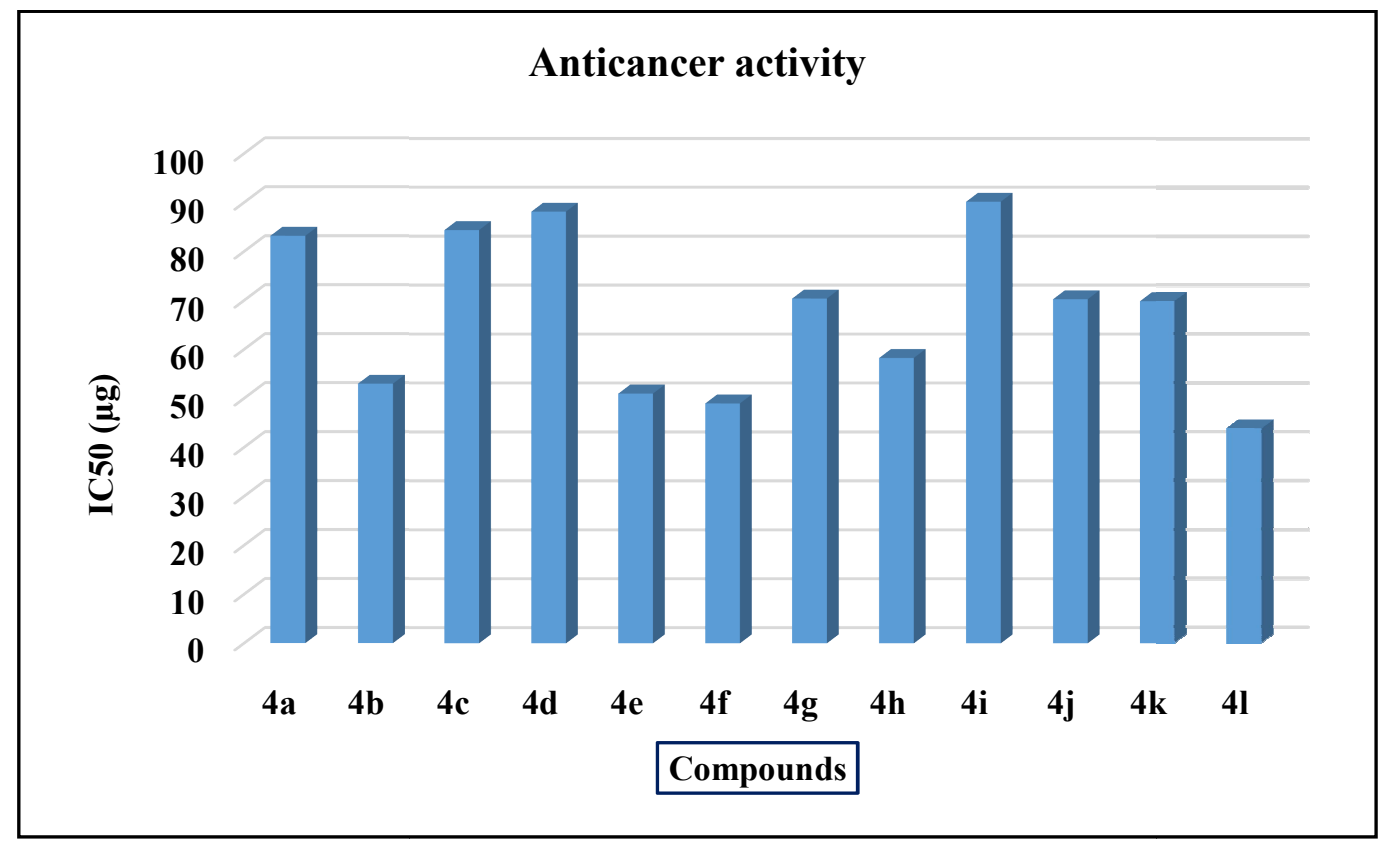

Figure 3: Graphical representation of anticancer activity of novel indole compounds (4a-4I) 

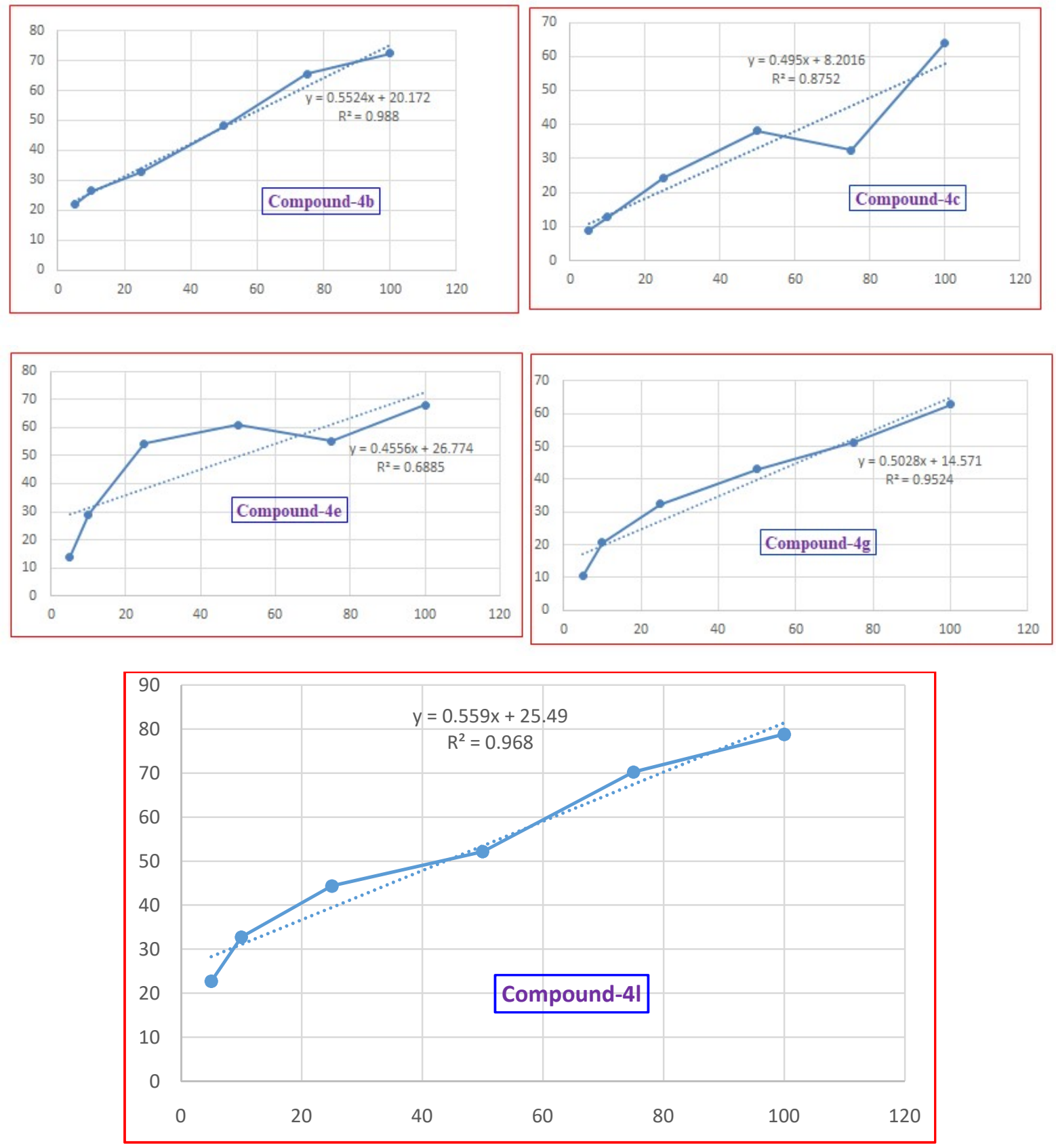

Figure 4: The cytotoxic activities of the synthesized indole derivatives against breast MCF7 cancer cell line

Table 4: Insilico EGFR Inhibition of Novel Indole Derivatives-Glide dock sores of the dataset ligands

\begin{tabular}{|c|c|c|c|c|c|c|}
\hline Compound No & $\begin{array}{l}\text { Dock score } \\
\text { XP GScore } \\
\end{array}$ & $\begin{array}{l}\text { No of H- } \\
\text { bonds }\end{array}$ & $\begin{array}{l}\text { Interacting } \\
\text { amino acids }\end{array}$ & $\begin{array}{l}\text { H-bond lengths } \\
(\AA)\end{array}$ & $\begin{array}{l}\text { Emodel } \\
\text { energy }\end{array}$ & Glide energy \\
\hline $4 \mathrm{c}$ & -5.861 & 3 & $\begin{array}{l}\text { ALA 88 } \\
\text { GLN } 119 \\
\end{array}$ & $\begin{array}{l}2.11,1.99 \\
2.17 \\
\end{array}$ & -60.578 & -38.599 \\
\hline 41 & -5.032 & 3 & $\begin{array}{l}\text { ALA 88 } \\
\text { GLN } 119 \\
\end{array}$ & $\begin{array}{l}2.64,2.00 \\
2.00\end{array}$ & -65.678 & -41.979 \\
\hline $4 k$ & -4.825 & 3 & $\begin{array}{l}\text { ALA 88 } \\
\text { GLN } 119\end{array}$ & $\begin{array}{l}2.47,1.98 \\
2.29\end{array}$ & -53.043 & -38.509 \\
\hline $4 f$ & -2.315 & 1 & TRP 143 & 1.81 & -53.837 & -37.222 \\
\hline $4 i$ & -2.261 & 1 & PRO 16 & 2.14 & -63.732 & -46.504 \\
\hline
\end{tabular}




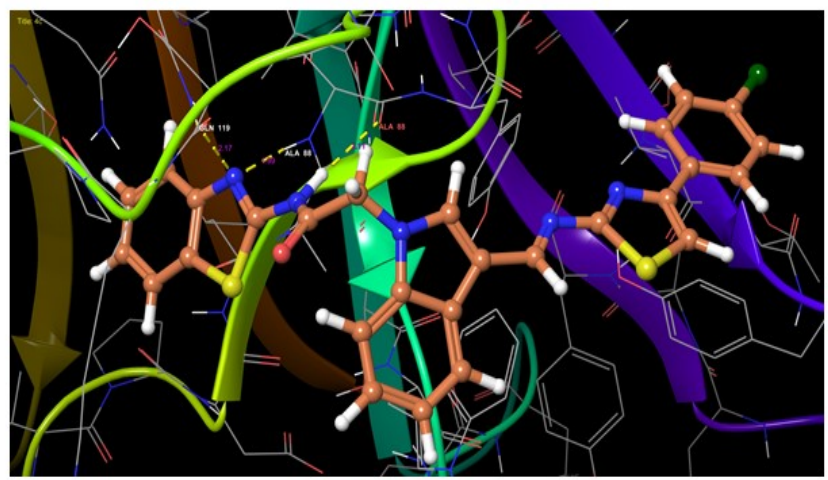

$4 \mathrm{c}$ docking-1

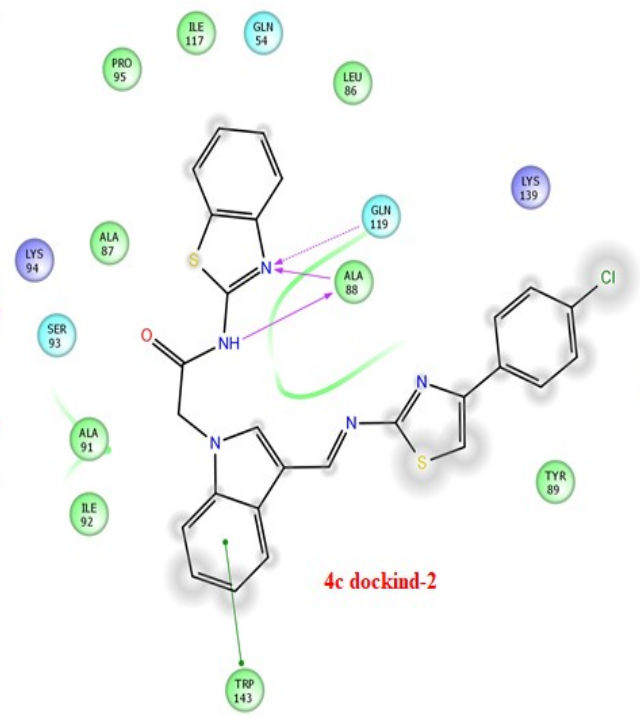

(i:ii:

(iBis
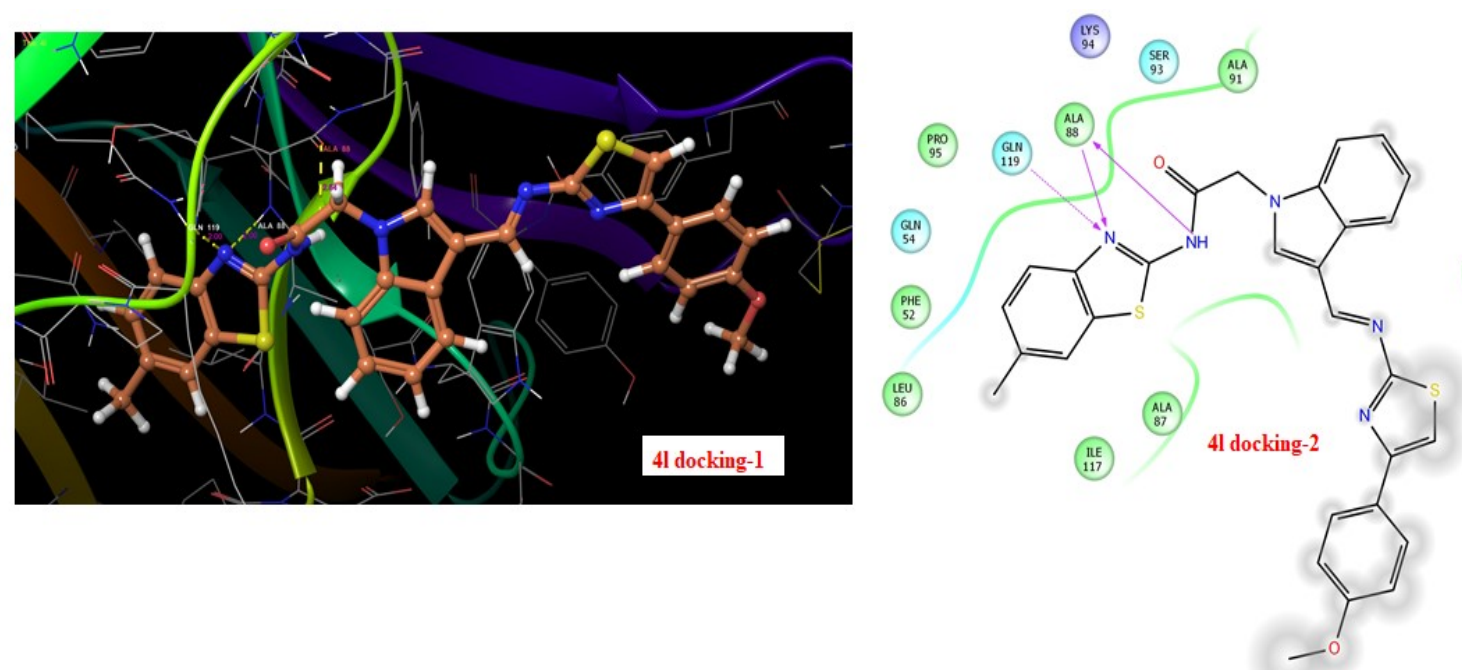

(ii:

Figure 5: Molecular docking structures of Indole derivatives (Docking Pose between the Ligand and the Protein)

\section{CONCLUSION}

A series of novel contain derivatives was performed and structures of the compounds were established by means of IR, ${ }^{1} \mathrm{HNMR}$ and mass spectral data as well as elemental analysis. All the synthesized compounds were screened to antibacterial, anticancer activities. In addition to molecular docking studies was performed to depict the essential structural components contributing to their pharmacological activity. Most of the compounds like $\mathbf{4 c}, \mathbf{4 d}, \mathbf{4 f}$ and $\mathbf{4 k}$ are showed potent antibacterial activity against all the tested bacterial strains and $\mathbf{4 b}, \mathbf{4 e}$ and $\mathbf{4 l}$ are the most active analogs in this study and showing broad spectrum activity toward the tested anticancer cell lines. Docking scores of all the synthesized novel indole deivatives were ranged between -2.261 to 5.861 .

\section{REFERENCES}

[1] Deng XQ, Song MX, Wei CX, et al. (2010). Synthesis and anticonvulsant activity of 7-alkoxytriazolo-[3, 4-b]benzo[d] thiazoles. Med Chem; 6:313-20. 
[2] Amnerkar ND, Bhusari KP. (2010). Synthesis, anticonvulsant activity and 3D-QSAR study of some prop2-eneamido and 1-acetyl-pyrazolin derivatives of aminobenzo [d] isothiazole. Eur J Med Chem; 45:149-59.

[3] Sreenivasa GM, Jayachandran E, Shivakumar B, et al. (2009). Synthesis of bioactive molecule fluoro benzo [d] isothiazole comprising potent heterocyclic moieties for anthelmentic activity. Arch. Peharm. Res.; 1: 150-7.

[4] Choi MM, Kim EA, Hahn HG, et al. (2007). Protective effect of benzo[d]isothiazole derivative KHG21834 on amyloid betainduced neurotoxicity in PC12 cells and cortical and mesencephalic neurons. Toxicology; 239: 156-66.

[5] T. Jiang, KL. Kuhen, K. Wolff, H. Yin, K. Bieza, J. Caldwell, B. Bursulaya, T. Tuntland, K. Zhang, D. Karanewsky, Y. H, Design, synthesis, and biological evaluations of novel oxindoles as HIV-1 non-nucleoside reverse transcriptase inhibitors. Part 2, Bioorg. Med. Chem. Lett., 16 (2006) 2109-2112.

[6] M. Verma, S. Nath Pandeya, K. Nand Singh, JP. Stables, Anticonvulsant activity of Schiff bases of isatin derivatives, Acta Pharm., 54 (2004) 49-54.

[7] A. Amal Raj, R. Raghunathan, MR. Sridevikumaria, N. Raman, Synthesis, antimicrobial and antifungal activity of a new class of spiro pyrrolidines, Bioorg. Med. Chem., 11 (2003) 407-419.

[8] R. Tripathy, A. Reiboldt, PA. Messina, M. Iqbal, J. Singh, ER. Bacon, TS. Angeles, SX. Yang, MS. Albom, C. Robinson, H. Chang, BA. Ruggeri, JP. Mallamo, Structure-guided identification of novel VEGFR-2 kinase inhibitors via solution phase parallel synthesis, Bioorg. Med. Chem. Lett., 16 (2006) 2158-2162.

[9] T. Ratan Bal, B. Anand, P. Yogeeswari, DH. Sriram, Synthesis and evaluation of anti-HIV activity of isatin $\beta$-thiosemicarbazone derivatives, Bioorg. Med. Chem. Lett. 15 (2005) 4451- 4455.

[10] G. Cecile, D. Douguet, V. Huteau, M. Gilles, ML. Helene, P. Sylvie, Substituted benzylpyrimidines targeting thymidine monophosphate kinase of Mycobacterium tuberculosis: synthesis and in vitro anti-mycobacterial activity, Bioorg. Med. Chem. 16 (2008) 6075-6085. 
[11] R. Lin, G. Sigmond, PJ. Johnson, SK. Connolly, E. Wetter, TV. Binnun, WV. Hughes, NB. Murray, SJ. Pandey, MM. Mazza, AR. Adams, F. Pesquera, AM. Steven, Synthesis and evaluation of 2, 7-diamino-thiazolo [4, 5-d] pyrimidine analogues as antitumor epidermal growth factor receptor (EGFR) tyrosine kinase inhibitors, Bioorg. Med. Chem. Lett., 19 (2009) 2333-2337.

[12] Q. Chen, X. Zhu, L. Jiang, LM. Yang, G. Fu, Synthesis, antifungal activity and CoMFA analysis of novel 1, 2, 4-triazolo [1, 5-a] pyrimidine derivatives, Eur. J. Med. Chem., 43 (2008) 595-603.

[13] MMM. Santos, Recent advances in the synthesis of biologically active spirooxindoles, Tetrahedron, 70 (2014) 9735-9757. [11] MA. Borad, MN. Bhoi, NP. Prajapati, HD. Patel, A Review on Synthesis of Spiro Heterocyclic Compounds from Isatin, Synth. Commun., 44 (2014) 897-922.

[14] MA. Borad, MN. Bhoi, NP. Prajapati, HD. Patel, A Review on Synthesis of multi Spiro Heterocyclic Compounds from Isatin, Synth. Commun., 44, (2014) 1-15.
[15] MA. Borad, MN. Bhoi, DP. Rajani, SD. Rajani, HD. Patel, synthesis and antimicrobial activities of 2-(4-substituted phenyl)-

1,10bdihydrospiro[benzo[e]pyrazo lo[1,5-c][1,3]oxazine-5, 3'indolin]-2'-one derivative from isatin, WJPPS, 3 (2014) 805-821.

[16] A. Agarwal, R. Ashutosh, N. Goyal, MS. Chauhana, S. Gupta, Dihydropyrido $\quad[2, \quad 3-d]$ pyrimidines as a new class of antileishmanial agents, Bioorg. Med. Chem. 13 (2005) 6678-6684.

[17] Selvam P, Chandramohan $M$, Clercq ED, Witvrouw M, Pannecouque C (2001) Synthesis and anti-HIV activity of $4-[(1,2-$ dihydro-2-oxo-3H-indol-3ylidene) amino]-N (4, 6-dimethyl2-pyrimidinyl)-benzene sulfonamide and itsderivatives. Eur J Pharm Sci 14:313-316

[18] Che Z, Tian Y, Liu S, Hu M, Chen G (2018) Discovery of Narylsulfonyl-3-acylindole benzoyl hydrazone derivatives as antiHIV-1 agents. Brazilian J Pharmaceutical Sci. 54(3) e17044: 1-7.

[19] Ashok P, Lu CL, Chander S, Zheng YT, Murugesan S (2015) Design, synthesis, and biological 
evaluation of 1-(thiophen-2-yl)-

9H-pyrido [3, 4-b] indole

derivatives as anti-HIV-1 agents.

Chem Biol Drug Des 85(6): 722

728

[20] Han X, Wu H, Wang W, Dong C,

Tien P, Wu S, Zhou HB (2014)

Synthesis and SARs of indole-

based $\alpha$-amino acids as potent

HIV-1 non-nucleoside reverse

transcriptase inhibitors. Org

Biomol Chem 12(41): 8308-8317. 